\title{
GENERAL METHODS OF CONSTRUCTING EQUIVALENT MINIMAL PAIRS NOT UNIQUE UP TO TRANSLATION
}

\author{
J. GRZYBOWSKI, S. KACZMAREK \\ and R. URBAŃSKI
}

\begin{abstract}
In this paper we give general methods of construction of various equivalent minimal pairs of compact convex sets that are not translates of one another.
\end{abstract}

\section{Introduction and preliminary remarks}

Let $X$ be a topological vector space; $\mathcal{K}(X)$ be the family of all non-empty compact convex subsets of $X$, and $\mathcal{K}^{2}(X)=\mathcal{K}(X) \times \mathcal{K}(X)$. Let

$$
A+B:=\{a+b \mid a \in A, b \in B\}
$$

be the Minkowski sum of $A$ and $B$ in $\mathcal{K}(X)$. The equivalence relation between pairs of compact convex sets is given by the relation $(A, B) \sim$ $(C, D)$ if and only if $A+D=B+C$. A partial order is given by the relation: $(A, B) \leq(C, D)$ if and only if $(A, B) \sim(C, D)$ and $A \subseteq C, B \subseteq$ $D$. By $[A, B]$, we denote the equivalence class of $(A, B)$ in $\mathcal{K}^{2}(X) / \sim$. The pair $(A, B) \in \mathcal{K}^{2}(X)$ is called minimal, if for all pairs $\left(A^{\prime}, B^{\prime}\right) \sim$ $(A, B)$ the inclusions $A^{\prime} \subset A$, and $B^{\prime} \subset B$ imply $A^{\prime}=A$ and $B^{\prime}=B$. For every $(A, B) \in \mathcal{K}^{2}(X)$ there exists a minimal element $\left(A^{\prime}, B^{\prime}\right)$ that is equivalent to $(A, B)$ and $A^{\prime} \subset A, B^{\prime} \subset B$ [4]. If $\operatorname{dim} X \leq 2$, the minimal element is unique up to translation [1],[10] i.e. if $(A, B)$ is minimal and $\left(A^{\prime}, B^{\prime}\right)$, equivalent to $(A, B)$, is also minimal, then there exists $x \in X$ such that $A=A+x$ and $B=B+x$. For $\operatorname{dim} X \geq 3$ there was given an example of equivalent minimal pairs not unique up to translation [1]. In

1991 Mathematics Subject Classification: 52A07, 26A27.

Servicio de Publicaciones. Universidad Complutense. Madrid, 2000 
[6] for the 3-dimensional case a continuum of equivalent minimal pairs wich are not connected by translation was constructed.

Now, in this paper, we give more general methods of constructing various (not necessarily polytops) equivalent minimal pairs not unique up to translation.

Let $X$ be a real topological vector space, $f \in X^{*}$ be a continuous linear functional, and $K \subseteq X$ a nonempty compact convex set. Then we denote by $H_{f}(K):=\left\{z \in K \mid f(z)=\max _{y \in K} f(y)\right\}$ the face of $K$ with respect to $f$. For the sum of two nonempty compact convex sets $A, B \subseteq X$ and $f \in X^{*}$ the following identity holds: $H_{f}(A+B)=H_{f}(A)+H_{f}(B)$. We will use the notation: $A \vee B:=\operatorname{conv}(A \cup B), A-B:=\{x \mid x+B \subset A\}$. In [7], by Pinsker, it was shown that for a locally convex topological vector space $X$ if $A, B, C \subseteq \mathcal{K}(X)$ then $(A \vee B)+C=(A+C) \vee(B+C)$. In [9], we can find for $A, B \subseteq \mathcal{K}(X)$. If $A \cup B$ is convex then $A+B=A \vee B+A \cap B$.

\section{First manner of constructing of equivalent min- imal pairs not unique up to translation}

Theorem 2.1. Let $A, A_{1}, P, Q \in \mathcal{K}(X), x \in X$ and assume that $A \cup P$, $A \cup Q, A \cup P \cup Q, A_{1} \cup P, A_{1} \cup(Q+x), A_{1} \cup P \cup(Q+x)$ are convex sets. Moreover, let $A \cap P=A_{1} \cap P,(A \cap Q)+x=A_{1} \cap(Q+x)$. Then $(A, B)$ is equivalent to $\left(A_{1}, B_{1}\right)$ where $B=A \cup P \cup Q$ and $B_{1}=A_{1} \cup P \cup(Q+x)$.

Proof. The sets $A \cup P, A \cup Q$ are convex. Hence, we have

$$
A+P=A \cup P+A \cap P,
$$


J. GRZYBOWSKI, S. KACZMAREK AND R. URBAŃSKI GENERAL METHODS OF ...

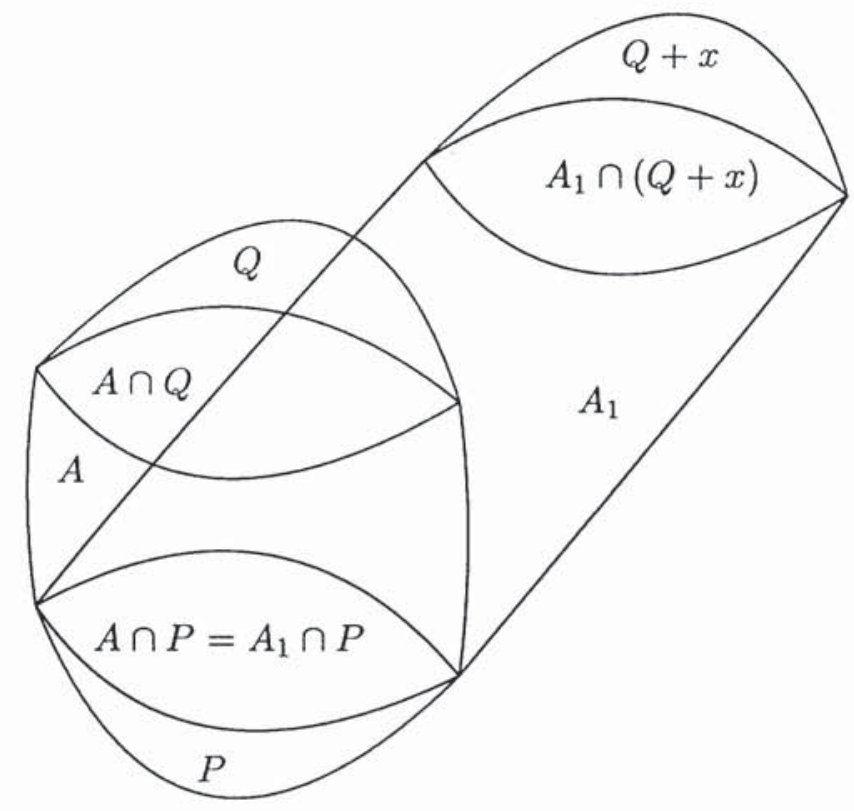

$$
A+Q=A \cup Q+A \cap Q .
$$

Analogously for $A_{1} \cup P$ and $A_{1} \cup(Q+x)$

$$
\begin{gathered}
A_{1}+P=A_{1} \cup P+A_{1} \cap P, \\
A_{1}+(Q+x)=A_{1} \cup(Q+x)+A_{1} \cap(Q+x) .
\end{gathered}
$$

From this it follows that

$$
\begin{gathered}
A+P+A_{1} \cup P+A_{1} \cap P=A \cup P+A \cap P+A_{1}+P, \\
A+Q+A_{1} \cup(Q+x)+A_{1} \cap(Q+x)=A \cup Q+A \cap Q+A_{1}+Q+x .
\end{gathered}
$$

Now, from the ordered law of cancellation we obtain

$$
\begin{gathered}
A+\left(A_{1} \cup P\right)=A \cup P+A_{1}, \\
A+A_{1} \cup(Q+x)=A \cup Q+A_{1} .
\end{gathered}
$$


Therefore from the Pinsker rule

$$
A+\left(A_{1} \cup P\right) \vee\left[A_{1} \cup(Q+x)\right]=A_{1}+A \cup P \cup Q .
$$

Hence

$$
A+B_{1}=A_{1}+B
$$

Suppose that $F, F_{1}, G, G_{1} \subset \mathcal{K}(X)$ and $F, F_{1}, G, G_{1} \subset f^{-1}(0)$ for some $f \in X^{*}$. Denote $P=F \vee\left(F_{1}+x_{1}\right), Q=\left(G+x_{2}\right) \vee\left(G_{1}+x_{3}\right)$, where $x_{1}, x_{2}, x_{3} \in X$ with $f\left(x_{1}\right)<0<f\left(x_{2}\right)<f\left(x_{3}\right)$.

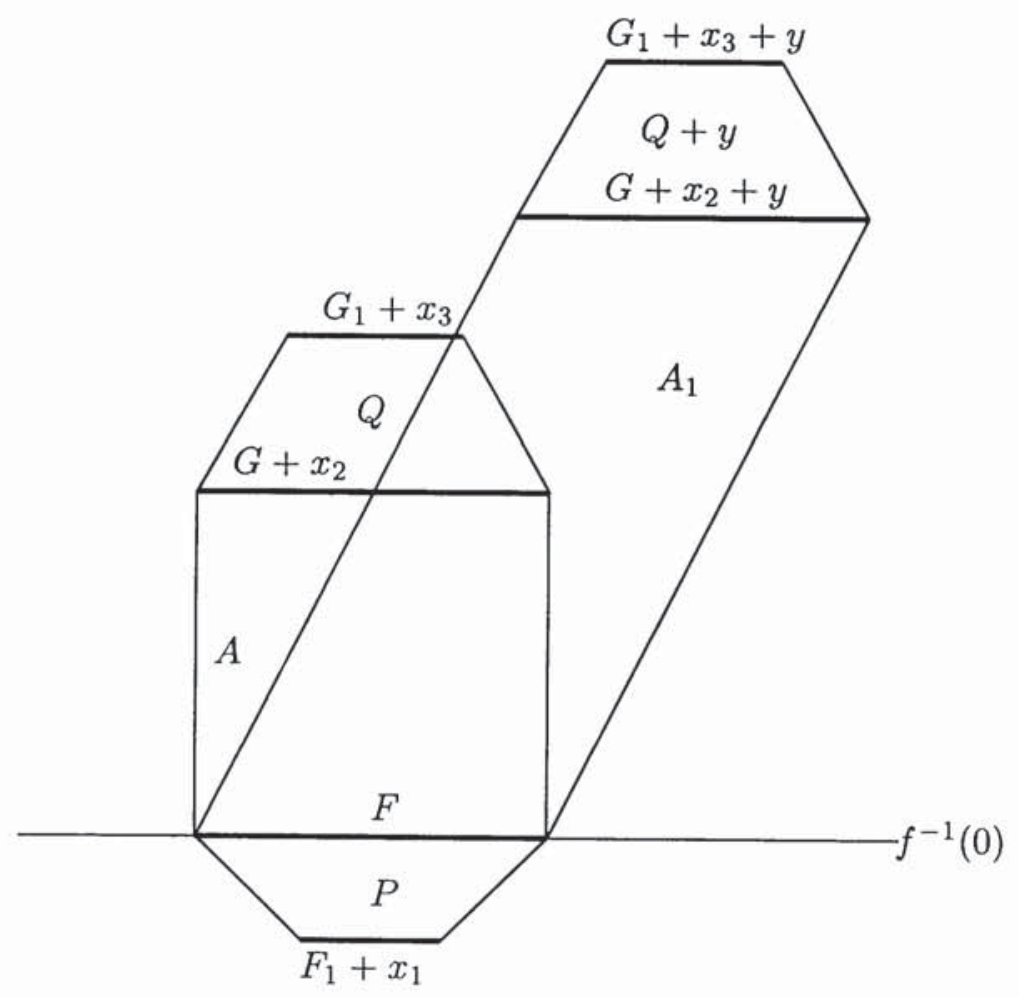

Corollary 2.2. Let $A=F \vee\left(G+x_{2}\right), B=P \vee Q, A_{1}=F \vee\left(G+y+x_{2}\right)$, $B_{1}=P \vee(Q+y), y \in X$ and

$$
f\left(x_{2}\right)\left(F_{1}+x_{1}\right)-f\left(x_{1}\right)\left(G+x_{2}\right) \subset f\left(x_{2}-x_{1}\right) F,
$$




$$
\begin{gathered}
f\left(x_{3}-x_{2}\right) F+f\left(x_{2}\right)\left(G_{1}+x_{3}\right) \subset f\left(x_{3}\right)\left(G+x_{2}\right) \\
f\left(x_{2}+y\right)\left(F_{1}+x_{1}\right)-f\left(x_{1}\right)\left(G+x_{2}+y\right) \subset f\left(x_{2}+y-x_{1}\right) F \\
f\left(x_{3}-x_{2}\right) F+f\left(x_{2}+y\right)\left(G_{1}+x_{3}+y\right) \subset f\left(x_{3}+y\right)\left(G+x_{2}+y\right) .
\end{gathered}
$$

Then $(A, B)$ is equivalent to $\left(A_{1}, B_{1}\right)$.

Corollary 2.2 follows immediately from Theorem 2.1 . Now, we define:

$$
\begin{gathered}
\mathcal{M}:=\left\{(A, B) \in \mathcal{K}^{2}(X) \mid(A, B) \text { is minimal }\right\}, \\
\mathcal{M}_{t}:=\left\{(A, B) \in \mathcal{M} \mid \forall\left(A^{\prime}, B^{\prime}\right) \in \mathcal{M},\left(A^{\prime}, B^{\prime}\right) \sim(A, B) \Rightarrow \exists x \in X,\right. \\
\left.A^{\prime}=A+x \text { and } B^{\prime}=B+x\right\}, \\
\mathcal{M}_{n t}:=\mathcal{M} \backslash \mathcal{M}_{t} .
\end{gathered}
$$

Theorem 2.3. Let $F, F_{1}, G, G_{1} \in \mathcal{K}(X), F, F_{1}, G, G_{1} \subset f^{-1}(0)$ for some $f \in X^{*}, A=F \vee\left(G+x_{2}\right), B=A \vee\left(F_{1}+x_{1}\right) \vee\left(G_{1}+x_{3}\right)$, where $f\left(x_{1}\right)<0<f\left(x_{2}\right)<f\left(x_{3}\right)$ and

$$
\begin{aligned}
& f\left(x_{2}\right)\left(F_{1}+x_{1}\right)-f\left(x_{1}\right)\left(G+x_{2}\right) \subset f\left(x_{2}-x_{1}\right) F, \\
& f\left(x_{3}-x_{2}\right) F+f\left(x_{2}\right)\left(G_{1}+x_{3}\right) \subset f\left(x_{3}\right)\left(G+x_{2}\right) .
\end{aligned}
$$

If $\left(F, F_{1}\right),\left(G, G_{1}\right) \in \mathcal{M}_{t}$ and $F \div G=G \div F=\emptyset$, then the pair $(A, B)$ is minimal.

Proof. Suppose that $\left(A^{\prime}, B^{\prime}\right) \leq(A, B)$ for some $\left(A^{\prime}, B^{\prime}\right) \in \mathcal{K}^{2}(X)$. Hence

$$
A+B^{\prime}=B+A^{\prime}
$$

From assumption there exists $f \in X^{*}$ such that

$$
H_{f} A=G+x_{2}, H_{f} B=G_{1}+x_{3}, H_{-f} A=F, H_{-f} B=F_{1}+x_{1} .
$$

Since we have

$$
H_{f} A+H_{f} B^{\prime}=H_{f} B+H_{f} A^{\prime}
$$

and

$$
H_{-f} A+H_{-f} B^{\prime}=H_{-f} B+H_{-f} A^{\prime} .
$$

We get

$$
G+x_{2}+H_{f} B^{\prime}=G_{1}+x_{3}+H_{f} A^{\prime}
$$


and

$$
F+H_{-f} B^{\prime}=F_{1}+x_{1}+H_{-f} A^{\prime}
$$

Hence

$$
\left(G+x_{2}, G_{1}+x_{3}\right) \sim\left(H_{f} A^{\prime}, H_{f} B^{\prime}\right)
$$

and

$$
\left(F, F_{1}+x_{1}\right) \sim\left(H_{-f} A^{\prime}, H_{-f} B^{\prime}\right) .
$$

Since $\left(G, G_{1}\right) \in \mathcal{M}_{t}$, then $G+x_{2}+z \subset H_{f} A^{\prime}$ and $G_{1}+x_{3}+z \subset H_{f} B^{\prime}$ for some $z \in X$. Therefore, $G+z+x_{2} \subset A$. Since $F-G=\emptyset$ and $A=F \vee\left(G+x_{2}\right)$ then $G+z+x_{2} \subset G+x_{2}$ and $z=0$. Hence $G+x_{2} \subset A^{\prime}$.

In similar way, taking $-f$ instead of $f$, we can prove that $F \subset A^{\prime}$.

Then $A=F \vee\left(G+x_{2}\right) \subset A^{\prime} \subset A$.

Hence $A^{\prime}=A$, and by the ordered law of cancellation $B^{\prime}=B$.

Corollary 2.4. Let us take sets described in Theorem 2.3. If there exists $y \in X, y \neq 0$ such that inclusions (2.1) and (2.2) are satisfied then Corollary 2.2 implies that $(A, B) \in \mathcal{M}_{n t}$.

The pair $\left(A_{1}, B_{2}\right)$ defined in Corollary 2.2 belongs $[A, B]$. Replacing $y$ by $\alpha y(\alpha \in(0,1))$ in definition of $\left(A_{1}, B_{1}\right)$ we obtain a pair in $[A, B]$. In fact, the set of all $y$ satisfying (2.1) and (2.2) is convex. Theorem 2.3 and Corollary 2.2 enable constructing great number of various pairs belonging to $\mathcal{M}_{n t}$. These pairs do not have to be polytops. In section 5 we present specific examples of pairs $(A, B)$ in $\mathcal{M}_{n t}$.

\section{The "flat" case}

It is interesting that $[A, B]$ containes a pair $(C, D)$ with "flat" $C$ i.e. such that $f(C)=\{0\}$. In this section we investigate that case.

Proposition 3.1. Let $A, B \in \mathcal{K}(X)$ be defined like in Theorem 2.3, $C=F+G, D=C \vee\left(F+G_{1}+x_{3}-x_{2}\right) \vee\left(G+F_{1}+x_{1}\right)$, $f\left(x_{3}\right)-f\left(x_{2}\right) \leq f\left(x_{2}\right),-f\left(x_{1}\right) \leq f\left(x_{2}\right)$,

$$
\left(f\left(x_{3}\right)-f\left(x_{2}\right)\right) F+f\left(x_{2}\right)\left(G_{1}+x_{3}\right) \subset f\left(x_{3}\right)\left(G+x_{2}\right)
$$


and

$$
-f\left(x_{1}\right)\left(G+x_{2}\right)+f\left(x_{2}\right)\left(F_{1}+x_{1}\right) \subset f\left(x_{2}-x_{1}\right) F .
$$

Then $(C, D)$ is equivalent to $(A, B)$.

Proof. We know that

$$
A=F \vee\left(G+x_{2}\right),
$$

and

$$
B=F \vee\left(F_{1}+x_{1}\right) \vee\left(G+x_{2}\right) \vee\left(G_{1}+x_{3}\right)
$$

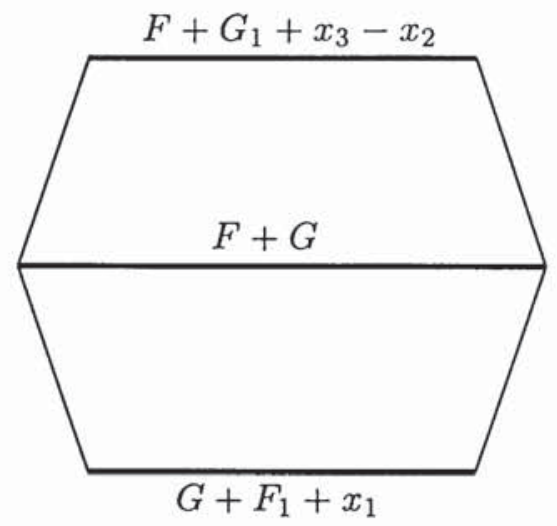

We have

$$
\begin{gathered}
A+D=(2 F+G) \vee\left(2 F+G_{1}+x_{3}-x_{2}\right) \vee\left(F+G+F_{1}+x_{1}\right) \vee \\
\vee\left(F+2 G+x_{2}\right) \vee\left(F+G+G_{1}+x_{3}\right) \vee\left(2 G+F_{1}+x_{1}+x_{2}\right), \\
B+C=(2 F+G) \vee\left(F+G+F_{1}+x_{1}\right) \vee\left(F+2 G+x_{2}\right) \vee\left(F+G+G_{1}+x_{3}\right) .
\end{gathered}
$$

Denote

$$
\alpha=\frac{f\left(x_{3}\right)-f\left(x_{2}\right)}{f\left(x_{2}\right)}, \beta=1-\alpha .
$$

Then

$$
\alpha F+G_{1}+x_{3} \subset(1+\alpha)\left(G+x_{2}\right) .
$$

Therefore,

$$
2 F+G_{1}+x_{3}-x_{2} \subset(1+\alpha)\left(G+x_{2}\right)+(1+\beta) F-x_{2}
$$


and

$$
2 F+G_{1}+x_{3}-x_{2} \subset(2 \alpha+\beta)\left(G+x_{2}\right)+(\alpha+2 \beta) F-x_{2} .
$$

We have

$$
2 F+G_{1}+x_{3}-x_{2} \subset \alpha\left(F+2 G+x_{2}\right)+\beta(2 F+G) .
$$

Analogously

$$
\alpha_{1}=\frac{-f\left(x_{1}\right)}{f\left(x_{2}\right)}, \quad \beta_{1}=1-\alpha_{1} .
$$

Then

$$
\alpha_{1}\left(G+x_{2}\right)+F_{1}+x_{1} \subset F\left(1+\alpha_{1}\right)
$$

hence

$$
2 G+F_{1}+x_{1}+x_{2} \subset\left(1+\beta_{1}\right) G+\beta_{1} x_{2}+F\left(1+\alpha_{1}\right) .
$$

So

$$
2 G+F_{1}+x_{1}+x_{2} \subset\left(\alpha_{1}+2 \beta_{1}\right) G+\beta_{1} x_{2}+F\left(2 \alpha_{1}+\beta_{1}\right),
$$

and

$$
2 G+F_{1}+x_{1}+x_{2} \subset \beta_{1}\left(F+2 G+x_{2}\right)+\alpha_{1}(2 F+G) .
$$

From (3.1) and (3.2) we have

$$
\begin{aligned}
& 2 F+G_{1}+x_{3}-x_{2} \subset\left(F+2 G+x_{2}\right) \vee(2 F+G), \\
& 2 G+F_{1}+x_{1}+x_{2} \subset\left(F+2 G+x_{2}\right) \vee(2 F+G) .
\end{aligned}
$$

Hence

$$
A+D=B+C \text {. }
$$

Let $A, B \in \mathcal{K}(X)$, then the following result holds:

Theorem 3.2. If $\left(F^{\prime}, G^{\prime}\right) \leq(F, G)$, then $(C, D)$ is equivalent to $\left(C^{\prime}, D^{\prime}\right)$, where $C^{\prime}=F+G^{\prime}, D^{\prime}=C^{\prime} \vee\left(F^{\prime}+G_{1}+x_{3}-x_{2}\right) \vee\left(G^{\prime}+F_{1}+x_{1}\right)$, $C=F+G$ and $D=C \vee\left(F+G_{1}+x_{3}-x_{2}\right) \vee\left(G+F_{1}+x_{1}\right)$.

Proof. We have $C=F+G$,

$C+D^{\prime}=\left(2 F+G+G^{\prime}\right) \vee\left(F+G+F^{\prime}+G_{1}+x_{3}-x_{2}\right) \vee\left(F+G+G^{\prime}+F_{1}+x_{1}\right)$ 
and

$$
\begin{gathered}
D+C^{\prime}=\left(F+G+F+G^{\prime}\right) \vee\left(F+F+G^{\prime}+G_{1}+x_{3}-x_{2}\right) \vee\left(F+G^{\prime}+G+F_{1}+x_{1}\right)= \\
\begin{array}{c}
=\left(2 F+G+G^{\prime}\right) \vee\left(F+G+F^{\prime}+G_{1}+x_{3}-x_{2}\right) \vee\left(F+G^{\prime}+G+F_{1}+x_{1}\right)= \\
=C+D^{\prime}
\end{array}
\end{gathered}
$$

Theorem 3.3. Let $\left(F^{\prime}, G^{\prime}\right) \leq(F, G)$ and $\left(F^{\prime}+G_{1}, G^{\prime}+F_{1}\right) \in \mathcal{M}_{t}$, then $\left(C^{\prime}, D^{\prime}\right)$ from Theorem 3.2 is also minimal pair.

Proof. Suppose that $\left(A^{\prime}, B^{\prime}\right) \leq\left(C^{\prime}, D^{\prime}\right)$. We have

$$
A^{\prime}+D^{\prime}=B^{\prime}+C^{\prime}
$$

and

$$
\begin{aligned}
H_{f} A^{\prime}+H_{f} D^{\prime} & =H_{f} B^{\prime}+H_{f} C^{\prime}, \\
H_{-f} A^{\prime}+H_{-f} D^{\prime} & =H_{-f} B^{\prime}+H_{-f} C^{\prime} .
\end{aligned}
$$

Then

$$
\begin{gathered}
A^{\prime}+F^{\prime}+G_{1}+x_{3}-x_{2}=H_{f} B^{\prime}+F^{\prime}+G, \\
A^{\prime}+G^{\prime}+F_{1}+x_{1}=H_{-f} B^{\prime}+F+G^{\prime} .
\end{gathered}
$$

Hence

$$
\begin{gathered}
A^{\prime}+G_{1}+x_{3}-x_{2}=H_{f} B^{\prime}+G, \\
A^{\prime}+F_{1}+x_{1}=H_{-f} B^{\prime}+F .
\end{gathered}
$$

Therefore

$$
A^{\prime}+G_{1}+x_{3}-x_{2}+H_{-f} B^{\prime}+F=A^{\prime}+F_{1}+x_{1}+H_{f} B^{\prime}+G .
$$

From the ordered law of cancellation we obtain

$$
G_{1}+F+H_{-f} B^{\prime}+x_{3}-x_{2}=G+F_{1}+H_{f} B^{\prime}+x_{1} .
$$

Hence

$$
\left(F+G_{1}, G+F_{1}\right) \sim\left(H_{f} B^{\prime}+x_{1}, H_{-f} B^{\prime}+x_{3}-x_{2}\right) .
$$


By assumption $\left(F^{\prime}+G_{1}, G^{\prime}+F_{1}\right) \in \mathcal{M}_{t}$.

Hence

$$
\begin{gathered}
F^{\prime}+G_{1}+x \subset H_{f} B^{\prime}+x_{1}, \\
G^{\prime}+F_{1}+x \subset H_{-f} B^{\prime}+x_{3}-x_{2} \text { for some } x \in X .
\end{gathered}
$$

From (3.3) it follows that

$$
A^{\prime}+G_{1}+x_{3}-x_{2} \supset F^{\prime}+G_{1}+x-x_{1}+G .
$$

Therefore,

$$
A^{\prime} \supset F^{\prime}+G+x-x_{1}+x_{2}-x_{3}=C^{\prime}+x-x_{1}+x_{2}-x_{3} .
$$

Since $C^{\prime} \subset A^{\prime}$ then $x-x_{1}+x_{2}-x_{3}=0$ and $A^{\prime}=C^{\prime}$. From the ordered law of cancellation, we have $B^{\prime}=D^{\prime}$.

Even if $\left(F^{\prime}+G_{1}, G^{\prime}+F_{1}\right) \notin \mathcal{M}_{t}$ we know that there exists a minimal pair $\left(C^{\prime}, D^{\prime}\right) \leq(C, D)[4]$. It means that $[A, B]$ contains minimal pair $\left(C^{\prime}, D^{\prime}\right)$ with "flat" $C^{\prime}$.

Proposition 3.4. Let $M, N \in \mathcal{K}(X),(S, T) \in \mathcal{M}_{t}, f \in X^{*}, H_{f} M=M$ and $H_{f} N=S, H_{-f} N=T$. Then for every $\left(M^{\prime}, N^{\prime}\right) \sim(M, N)$ such that $H_{f} M^{\prime}=M^{\prime}$ and $\left(M^{\prime}, N^{\prime}\right)$ is minimal we have $M^{\prime}=M+x, N^{\prime}=N+x$ for some $x \in X$.

Proof. Let $M^{\prime}+N=N^{\prime}+M$. Then

$$
\begin{gathered}
M^{\prime}+S=H_{f} N^{\prime}+M, \\
M^{\prime}+T=H_{-f} N^{\prime}+M .
\end{gathered}
$$

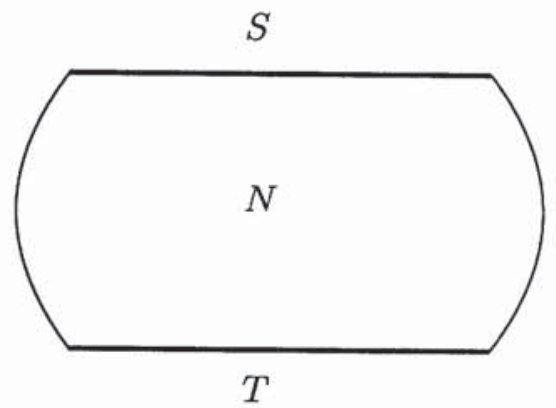


Hence

$$
M^{\prime}+S+H_{-f} N^{\prime}+M=M^{\prime}+T+H_{f} N^{\prime}+M .
$$

Then

$$
S+H_{-f} N^{\prime}=T+H_{f} N^{\prime},
$$

and we have

$$
(S, T) \sim\left(H_{f} N^{\prime}, H_{-f} N^{\prime}\right) .
$$

But $(S, T) \in \mathcal{M}_{t}$. Therefore

$$
S+x \subset H_{f} N^{\prime}, T+x \subset H_{-f} N^{\prime} \quad \text { for some } x \in X .
$$

Now, from (3.5) we have

$$
M^{\prime}+S \supset S+M+x .
$$

This implies that $M^{\prime} \supset M+x$. From $M^{\prime}+N=N^{\prime}+M$, we obtain $N+x \subset N^{\prime}$. But $\left(N^{\prime}, M^{\prime}\right)$ is minimal.

Hence $N^{\prime}=N+x$ and $M^{\prime}=M+x$.

Corollary 3.5. Let $\left(C^{\prime}, D^{\prime}\right)$ be defined like in Theorem 3.3. For every $\left(C_{1}, D_{1}\right) \sim\left(C^{\prime}, D^{\prime}\right)$ such that $H_{f} C_{1}=C_{1}$ and $\left(C_{1}, D_{1}\right)$ is minimal there exists $x \in X$ such that $C_{1}=C^{\prime}+x$ and $D_{1}=D^{\prime}+x$. Then $\left(C^{\prime}, D^{\prime}\right)$ is unique up to translation minimal pair in $[A, B]$ that has "flat" $C^{\prime}$.

\section{Second manner of constructing equivalent min- imal pairs not unique up to translation}

Let $A, B$ be defined like in Theorem 2.1 and $C=A \cup P, D=A \cup Q$. The pair $(C, D)$ is not equivalent to $(A, B)$, but the pair $(C, D)$ opens new possibility of construction equivalent minimal pairs not unique up to translation.

Theorem 4.1. Let $A, A_{1}, P, Q$ be defined like in Theorem 2.1. Then $(C, D)$ is equivalent to $\left(C_{1}, D_{1}\right)$ where $C=A \cup P, D=A \cup Q, C_{1}=$ $A_{1} \cup P$ and $D_{1}=A_{1} \cup(Q+x)$.

Proof. The sets $A \cup P, A \cup Q$ are convex. Hence

$$
A+P=A \cup P+A \cap P,
$$




$$
A+Q=A \cup Q+A \cap Q .
$$

Analogously for $A_{1} \cup P$ and $A_{1} \cup(Q+x)$

$$
\begin{gathered}
A_{1}+P=A_{1} \cup P+A_{1} \cap P, \\
A_{1}+(Q+x)=A_{1} \cup(Q+x)+A_{1} \cap(Q+x) .
\end{gathered}
$$

From above it follows

$$
\begin{gathered}
A+P+A_{1} \cup P+A_{1} \cap P=A_{1}+P+A \cup P+A \cup P+A \cap P, \\
A+Q+A_{1} \cup(Q+x)+A_{1} \cap(Q+x)=A_{1}+Q+x+A \cup Q+A \cap Q .
\end{gathered}
$$

Now from the ordered law of cancellation we obtain

$$
\begin{gathered}
A+A_{1} \cup P=A_{1}+A \cup P, \\
A+A_{1} \cup(Q+x)=A_{1}+A \cup Q .
\end{gathered}
$$

Therefore

$$
\begin{aligned}
A+A_{1} \cup P+A_{1}+A \cup Q & =A_{1}+A \cup P+A+A_{1} \cup(Q+x), \\
A_{1} \cup P+A \cup Q & =A \cup P+A_{1} \cup(Q+x) .
\end{aligned}
$$

Hence

$$
C_{1}+D=C+D_{1}
$$

Corollary 4.2. Let $A, A_{1}, P, Q$ be defined like in Corollary 2.2. Then $(C, D)$ is equivalent to $\left(C_{1}, D_{1}\right)$ where $C=A \vee P, D=A \vee Q, C_{1}=$ $A_{1} \vee P$ and $D_{1}=A_{1} \vee(Q+x)$.

Theorem 4.3. Let $F, F_{1}, G, G_{1} \in \mathcal{K}(X), F, F_{1}, G, G_{1} \subset f^{-1}(0)$ for some $f \in X^{*}, C=\left(F_{1}+x_{1}\right) \vee F \vee\left(G+x_{2}\right), D=F \vee\left(G+x_{2}\right) \vee\left(G_{1}+x_{3}\right)$ where $f\left(x_{1}\right)<0<f\left(x_{2}\right)<f\left(x_{3}\right)$ and

$$
\begin{gathered}
f\left(x_{2}\right)\left(F_{1}+x_{1}\right)-f\left(x_{1}\right)\left(G+x_{2}\right) \subset\left(f\left(x_{2}\right)-f\left(x_{1}\right)\right) F, \\
f\left(x_{3}-x_{2}\right) F+f\left(x_{2}\right)\left(G_{1}+x_{3}\right) \subset f\left(x_{3}\right)\left(G+x_{2}\right) .
\end{gathered}
$$

If $\left(F, F_{1}\right),\left(G, G_{1}\right) \in \mathcal{M}_{t}$ and $F \div G=G \div F=\emptyset$, then the pair $(C, D)$ is minimal. 
Proof. Suppose that $\left(C^{\prime}, D^{\prime}\right) \leq(C, D)$ for some $\left(C^{\prime}, D^{\prime}\right) \in \mathcal{K}^{2}(X)$. Hence

$$
C+D^{\prime}=D+C^{\prime} \text {. }
$$

From assumption there exists $f \in X^{*}$ such that $H_{f} C=G+x_{2}, H_{f} D=$ $G_{1}+x_{3}, H_{-f} C=F, H_{-f} D=F_{1}+x_{1}$. Since we have

$$
H_{f} C+H_{f} D^{\prime}=H_{f} D+H_{f} C^{\prime}
$$

and

$$
H_{-f} C+H_{-f} D^{\prime}=H_{-f} D+H_{-f} C^{\prime}
$$

Then

$$
G+x_{2}+H_{f} D^{\prime}=G_{1}+x_{3}+H_{f} C^{\prime}
$$

and

$$
F_{1}+x_{1}+H_{-f} D^{\prime}=F+H_{-f} C^{\prime} .
$$

Hence

$$
\left(G+x_{2}, G_{1}+x_{3}\right) \sim\left(H_{f} C^{\prime}, H_{f} D^{\prime}\right)
$$

and

$$
\left(F, F_{1}+x_{1}\right) \sim\left(H_{-f} D^{\prime}, H_{-f} C^{\prime}\right) .
$$

Since $\left(G, G_{1}\right) \in \mathcal{M}_{t}$, then $G+x_{2}+x \subset H_{f} C^{\prime}$ and $G_{1}+x_{3}+x \subset H_{f} D^{\prime}$ for some $x \in X$. Because $F-G=\emptyset$ and $C=\left(F_{1}+x_{1}\right) \vee F \vee\left(G+x_{2}\right)$ then $G+x_{2}+x \subset H_{f} C^{\prime} \subset G+x_{2}$ and $x=0$. Hence $H_{f} C^{\prime}=G+x_{2}$ and from the ordered law of cancellation $H_{f} D^{\prime}=G_{1}+x_{3}$. Similarly, taking $-f$ instead of $f$, we can prove that $H_{-f} C^{\prime}=F_{1}+x_{1}$ and $H_{-f} D^{\prime}=F$. Denote $C^{\prime \prime}=\left(F_{1}+x_{1}\right) \vee\left(G+x_{2}\right)$ and $D^{\prime \prime}=F \vee\left(G_{1}+x_{1}\right)$. We know that $C^{\prime \prime} \subset C^{\prime} \subset C$ and $D^{\prime \prime} \subset D^{\prime} \subset D$. We have

$$
C^{\prime \prime} \vee D^{\prime \prime}=\left(F_{1}+x_{1}\right) \vee F \vee\left(G+x_{2}\right) \vee\left(G_{1}+x_{3}\right)=C \vee D
$$

then $C^{\prime} \vee D^{\prime}=C \vee D$. Moreover $C+D^{\prime}=D+C^{\prime}$. This implies that $C+C^{\prime} \vee D^{\prime}=\left(C+C^{\prime}\right) \vee\left(C+D^{\prime}\right)=\left(C+C^{\prime}\right) \vee\left(D+C^{\prime}\right)=C^{\prime}+C \vee D$.

But $C^{\prime} \vee D^{\prime}=C \vee D$ and from the ordered law of cancellation, we obtain $C=C^{\prime}$ and $D=D^{\prime}$. 
Theorem 4.4. Let $(C, D)$ be defined like in Theorem 4.3, $M=\left(F_{1}+\right.$ $\left.G+x_{1}+x_{2}\right) \vee\left(F+G+x_{2}\right)$ and $N=\left(G+F+x_{2}\right) \vee\left(G_{1}+F+x_{3}\right)$. Then $(M, N)$ is equivalent to $(C, D)$.

Proof. We have $C=C_{1} \cup C_{2}$ and $D=D_{1} \cup D_{2}$, where $C_{1}=\left(F_{1}+x_{1}\right) \vee F$, $C_{2}=D_{1}=F \vee\left(G+x_{2}\right)$ and $D_{2}=\left(G+x_{2}\right) \vee\left(G_{1}+x_{3}\right)$.

Pairs $\left(C_{1}, C_{2}\right)$ and $\left(D_{1}, D_{2}\right)$ are convex. Hence

$$
C_{1}+C_{2}=C+F
$$

and

$$
D_{1}+D_{2}=D+G+x_{2} \text {. }
$$

Then

$$
C_{1}+C_{2}+D+G+x_{2}=D_{1}+D_{2}+C+F .
$$

Now, from the ordered law of cancellation, we obtain

$$
C_{1}+D+G+x_{2}=C+F+D_{2} .
$$

Therefore

$$
D+M=C+N \text {. }
$$

The sets $M, N$ are a special type of sets called general frustums. Criteria of minimality of pairs of frustums, can be found [3].

\section{$5 \quad$ Examples}

1). Let $G=\{(x, y, z) \mid z=0,-2 \leq x \leq 2,-1 \leq y \leq 1\}, F=$ $\{(x, y, z) \mid z=0,-1 \leq x \leq 1,-2 \leq y \leq 2\}, G_{1}=\{(0,0,0)\}, F_{1}=$ $\{(0,0,0)\} . x_{1}=(0,0,-1), x_{2}=(0,0,2), x_{3}=(0,0,3), y=(1,1,1)$. $P=F \vee\left(F_{1}+(0,0,-1)\right), Q=(G+(0,0,2)) \vee\left(G_{1}+(0,0,3)\right), A=$ $F \vee(G+(0,0,2)), B=P \vee Q, A_{1}=F \vee(G+(1,1,3)), B_{1}=P \vee(Q+$ $(1,1,1))$, Pairs $(A, B)$ and $\left(A_{1}, B_{1}\right)$ satisfy Theorem 2.3 i.e., $(A, B) \sim$ $\left(A_{1}, B_{1}\right)$ and $(A, B),\left(A_{1}, B_{1}\right) \in \mathcal{M}_{n t}$.

Take $F^{\prime}=\{0\} \times[-1,1] \times\{0\}$ and $G^{\prime}=[-1,1] \times\{0\} \times\{0\}$, then $\left(F^{\prime}, G^{\prime}\right) \leq$ $(F, G)$ and $\left(F^{\prime}+G_{1}, G^{\prime}+F_{1}\right) \in \mathcal{M}_{t}$. From Theorems 3.2 and 3.3 we have $C^{\prime}=F+G^{\prime}, D^{\prime}=C^{\prime} \vee\left(F^{\prime}+G_{1}+(0,0,1)\right) \vee\left(G^{\prime}+F_{1}+(0,0,-1)\right)$, $\left(C^{\prime}, D^{\prime}\right) \sim(A, B)$ and $(A, B),\left(C^{\prime}, D^{\prime}\right) \in \mathcal{M}_{n t}$. 
2). Let $F=\left\{(x, y, z) \mid z=0,(x+2)^{2}+y^{2} \leq 8,(x-2)^{2}+y^{2} \leq 8\right\}$, $G=\left\{(x, y, z) \mid z=0, x^{2}+(y+2)^{2} \leq 8, x^{2}+(y-2)^{2} \leq 8\right\}, F_{1}=$ $\{0\} \times[-1,1] \times\{0\}, G_{1}=[-1,1] \times\{0\} \times\{0\} . x_{1}=(0,0,-1), x_{2}=$ $(0,0,2), x_{3}=(0,0,3), y=(1,1,1) . P=F \vee\left(F_{1}+(0,0,-1)\right), Q=$ $(G+(0,0,2)) \vee\left(G_{1}+(0,0,3)\right), A=F \vee(G+(0,0,2)), B=P \vee Q$, $A_{1}=F \vee(G+(1,1,3)), B_{1}=P \vee(Q+(1,1,1))$. We have $(A, B) \sim$ $\left(A_{1}, B_{1}\right)$ and $(A, B),\left(A_{1}, B_{1}\right) \in \mathcal{M}_{n t}$. In this example $(F, G)$ satisfies Theorem 3.3 namely $\left(F+G_{1}, G+F_{1}\right) \in \mathcal{M}_{t} . C=F+G, D=C \vee(F+$ $\left.G_{1}+(0,0,1)\right) \vee\left(G+F_{1}+(0,0,-1)\right),(A, B) \sim(C, D)$ and $(A, B),(C, D) \in$ $\mathcal{M}_{n t}$.

\section{References}

[1] J. Grzybowski, On minimal pairs of convex compact sets, Archiv der Mathematik 63 (1994) 173-181.

[2] L. Hörmander, Sur la fonction d'appui des ensembles convex dans une espace localement convexe, Arkiv for Math. 3 (1954), 181-186.

[3] S. Kaczmarek, Minimal pairs in classes of frustums, Comm. Math. 39 (1999), 74-86.

[4] D. Pallaschke, S. Scholtes, R. Urbański, On Minimal Pairs of Compact Convex Sets, Bull. Polish. Acad. Sci. Math. 39 (1) (1991) 1-5.

[5] D. Pallaschke, R. Urbański, Reduction of Quasidifferentials and Minimal Representations, Math. Programing, Series A 66 (1994) 161-180.

[6] D. Pallaschke, R. Urbański, A Continuum of Minimal Pairs of Compact Convex Sets wich are not Connected by Translations, Journal of Convex Analysis 3 (1996) 83-95.

[7] A. G. Pinsker, The space of convex sets of locally convex space, Trudy Leningrad Engineering-Economic Institute, 63 (1966) 13-17.

[8] A. M. Rubinow, I. S. Akhundow, Differences of compact sets in the sense of Demianow and its application to non-smoth analysis, Optimiz.,23 (1992) 179-188.

[9] R. Schneider, Convex Bodies: The Brun-Minkowski Theory (Encyklopedia of Math. end its Appl.,Vol.44), Cambridge University Press, Cambridge 1993.

[10] S. Scholtes, Minimal pairs of convex bodies in two dimensions, Mathematika 39 (1992) 267-273. 
[11] R. Urbański, A generalization of the Minkowski-Rådström-Hörmander Theorem, Bull. Acad. Polon. Sci. Sér. Sci. Math. Astr. Phys. 24 (1976) 709-715.

Faculty of Mathematics and Computer Science

Adam Mickiewicz University

Matejki 48/49

60-769 Poznań, Poland

E-mail:

jgrz@amu.edu.pl

kacstan@amu.edu.pl

rich@amu.edu.pl

Recibido: 23 de Junio de 1999

Revisado: 13 de Marzo de 2000 